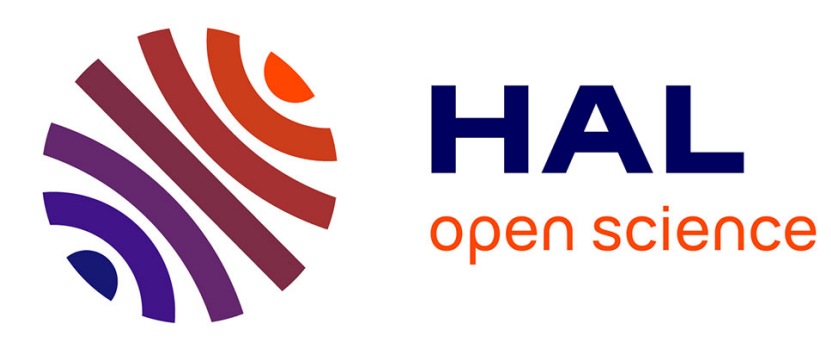

\title{
A wave-based reduction technique for the dynamic behavior of periodic structures
}

Denis Duhamel, Jean-Mathieu Mencik

\section{To cite this version:}

Denis Duhamel, Jean-Mathieu Mencik. A wave-based reduction technique for the dynamic behavior of periodic structures . COMPDYN 2015, May 2015, Hersonissos, Greece. hal-01266388

\section{HAL Id: hal-01266388 \\ https://hal.science/hal-01266388}

Submitted on 2 Feb 2016

HAL is a multi-disciplinary open access archive for the deposit and dissemination of scientific research documents, whether they are published or not. The documents may come from teaching and research institutions in France or abroad, or from public or private research centers.
L'archive ouverte pluridisciplinaire HAL, est destinée au dépôt et à la diffusion de documents scientifiques de niveau recherche, publiés ou non, émanant des établissements d'enseignement et de recherche français ou étrangers, des laboratoires publics ou privés. 


\title{
A WAVE-BASED REDUCTION TECHNIQUE FOR THE DYNAMIC BEHAVIOR OF PERIODIC STRUCTURES
}

\author{
Denis Duhamel ${ }^{1}$ and Jean-Mathieu Mencik ${ }^{2}$ \\ ${ }^{1}$ Université Paris-Est, Laboratoire Navier, ENPC/IFSTTAR/CNRS, 6 et 8 Avenue Blaise Pascal, Cité \\ Descartes, Champs-sur-Marne, 77455 Marne La Vallée Cedex 2, France \\ e-mail: denis.duhamel@enpc.fr \\ 2 INSA Centre Val de Loire, Université François Rabelais de Tours, LMR EA 2640, Campus de Blois, \\ 3 Rue de la Chocolaterie, CS 23410, 41034 Blois Cedex, France \\ e-mail: jean-mathieu.mencik@insa-cvl.fr
}

Keywords: Wave Finite Element Method, Periodic Structures, Model Reduction, Structural Dynamics.

\begin{abstract}
The wave finite element (WFE) method is investigated to describe the dynamic behavior of periodic structures like those composed of arbitrary-shaped substructures along a certain straight direction. A generalized eigenproblem based on the so-called $\mathbf{S}+\mathbf{S}^{-1}$ transformation is proposed for accurately computing the wave modes which travel in right and left directions along those periodic structures. Besides, a model reduction technique is proposed which involves partitioning a whole periodic structure into one central structure surrounded by two extra substructures. In doing so, a few wave modes are only required for modeling the central periodic structure. A comprehensive validation of the technique is performed on a $2 D$ periodic structure. Also, its efficiency in terms of CPU time savings is highlighted regarding a $3 D$ periodic structure that exhibits substructures with large-sized FE models.
\end{abstract}




\section{Introduction}

Predicting the dynamic behavior of complex periodic structures like those encountered in engineering applications, by means of accurate and fast numerical tools, constitutes an open industrial challenge. In many cases, these systems are made up of arbitrary-shaped substructures which are identical to each other and are assembled along one straight direction. The need to reduce the CPU times involved by the computation of the forced response of this kind of periodic structures relates the motivation of the present paper. This issue is addressed here by means of the wave finite element (WFE) combined with an original model reduction technique.

Originally, the WFE method has been initiated to describe the wave propagation along onedimensional periodic elastic systems [1,2]. The computation of the forced response of bounded elastic waveguides - i.e., periodic structures which are made up of straight substructures subject to Neumann and Dirichlet boundary conditions (BCs) has been addressed in several ways using the WFE method $[3,4,5,6]$. The procedure consists in assessing the kinematic and mechanical fields of a structure by means of numerical wave modes traveling in right and left directions. Their computation follows by considering a generalized eigenproblem that is expressed from the mass and stiffness matrices of a particular substructure. Actually, there exist two main WFE-based strategies to compute the forced response of periodic structures. These are labeled as dynamic stiffness matrix (DSM) approach and wave amplitudes (WA) approach, and respectively involve expressing the condensed dynamic stiffness matrix of a periodic structure in terms of wave modes [3], or the vectors of wave amplitudes of the right-going and left-going modes $[4,7,8]$. The feature of the WFE method is that it makes use of the FE model of one single substructure only, rather than considering the full FE model of a whole periodic structure. This makes the WFE method quite fast for describing the dynamic behavior of periodic structures when compared to the conventional FE method and FE-based CMS methods like the Craig-Bampton method [9].

Two features are addressed in this work. One feature is the use of a generalized eigenproblem based on the so-called $\mathbf{S}+\mathbf{S}^{-1}$ transformation to compute the wave modes with accurate precision. In this context, exact analytic relations can be considered so as to ensure the coherence between the right-going and left-going wave modes. Besides, the Lanczos method [10] is used to speed up the computation of the WFE eigenproblem with a view to calculating a reduced number of wave modes only. To achieve this task, an error indicator already proposed in [11] is considered to select in an a priori process the number of wave modes that need to be computed. As a second feature of this work, an efficient model reduction technique is proposed which consists in partitioning a whole periodic structure into one central structure surrounded by two extra substructures. In doing so, a few wave modes are only required for modeling the central periodic structure, hence enabling the computation of those wave modes to be achieved in a very fast way by means of the Lanczos method.

The rest of the paper is organized as follows. In Section 2, the basics of the WFE method for describing the wave propagation along a one-dimensional periodic structure are recalled. The strategy to compute the wave modes by means of a generalized eigenproblem based on the $\mathbf{S}+\mathbf{S}^{-1}$ transformation is proposed. In Section 3, the WFE-based model reduction technique, which consists in partitioning a whole periodic structure into one central structure surrounded by two extra substructures, is fully developed. Numerical experiments are finally brought in Section 4 that concern a 2D structure as well as a 3D stiffened shell with a periodic distribution of longitudinal and circumferential stiffeners. 


\section{WFE method}

\subsection{Basic framework}

The basics of the WFE method for describing waves which travel in right and left directions along a one-dimensional periodic structure (see Figure 1) are recalled hereafter.

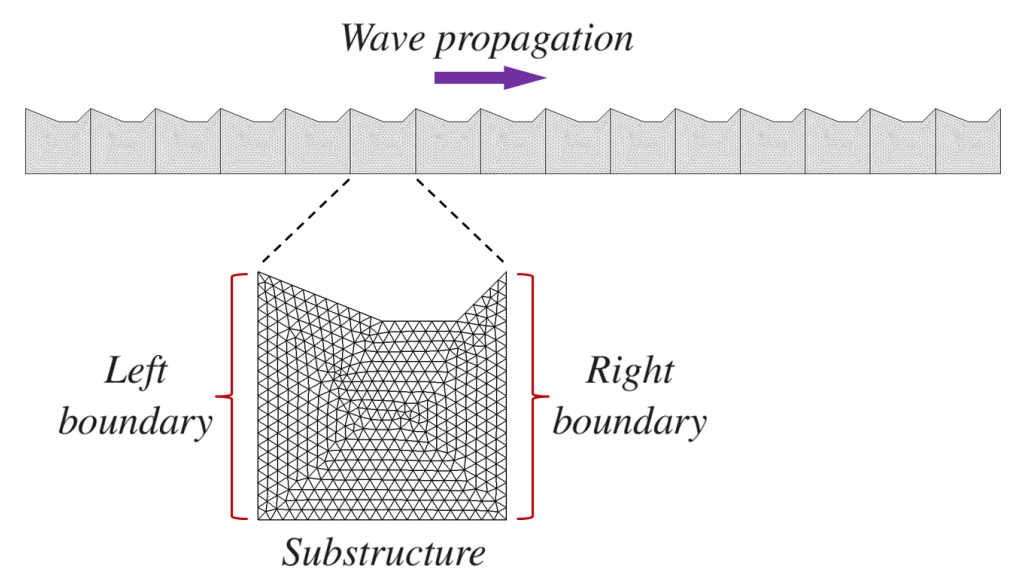

Figure 1: FE mesh of a periodic structure and related substructure.

Within the present framework, the structures are assumed to be linear, elastic and damped by means of a constant loss factor $\eta$. Also, they involve excitation sources which are assumed to be harmonic time-dependent, i.e., of the form $g(\omega) \exp (i \omega t)$ where $\omega$ denotes the pulsation. Besides, the substructures are modeled by means of the same FE mesh with a same number (say, $n$ ) of DOFs over their left and right boundaries. In matrix form, the dynamic equilibrium equation of a given substructure is expressed in the frequency domain as

$$
\mathrm{Dq}=\mathbf{F},
$$

where $\mathbf{D}$ is the dynamic stiffness matrix of the substructure. It is expressed as $\mathbf{D}=-\omega^{2} \mathbf{M}+$ $(1+i \eta) \mathbf{K}$ where $\mathbf{M}$ and $\mathbf{K}$ refer to the mass and stiffness matrices, respectively. Also in Eq. (1), $\mathbf{q}$ and $\mathbf{F}$ are, respectively, the vectors of nodal displacements/rotations and nodal forces/moments of the substructure. The key idea behind the WFE method is to express a relation which links the kinematic/mechanical quantities on the right boundary of the substructure to those on its left boundary. From Eq. (1), it is expressed as [12]:

$$
\mathbf{u}_{\mathrm{R}}=\mathbf{S u}_{\mathrm{L}}
$$

where $\mathbf{u}_{\mathrm{R}}$ and $\mathbf{u}_{\mathrm{L}}$ are $2 n \times 1$ state vectors, expressed as

$$
\mathbf{u}_{\mathrm{R}}=\left[\begin{array}{c}
\mathbf{q}_{\mathrm{R}} \\
\mathbf{F}_{\mathrm{R}}
\end{array}\right] \quad, \quad \mathbf{u}_{\mathrm{L}}=\left[\begin{array}{c}
\mathbf{q}_{\mathrm{L}} \\
-\mathbf{F}_{\mathrm{L}}
\end{array}\right] .
$$

In Eq. (3), the subscripts $L$ and $R$ denote the DOFs which belong to the left and right boundaries of the substructure, respectively. Also, $\mathrm{S}$ is a $2 n \times 2 n$ matrix expressed as

$$
\mathbf{S}=\left[\begin{array}{c|c}
-\mathbf{D}_{\mathrm{LR}}^{*-1} \mathbf{D}_{\mathrm{LL}}^{*} & -\mathbf{D}_{\mathrm{LR}}^{*-1} \\
\hline \mathbf{D}_{\mathrm{RL}}^{*}-\mathbf{D}_{\mathrm{RR}}^{*} \mathbf{D}_{\mathrm{LR}}^{*-1} \mathbf{D}_{\mathrm{LL}}^{*} & -\mathbf{D}_{\mathrm{RR}}^{*} \mathbf{D}_{\mathrm{LR}}^{*-1}
\end{array}\right],
$$


where $\mathbf{D}^{*}$ refers to the dynamic stiffness matrix of the substructure condensed on its left and right boundaries.

We are looking for wavemodes $\left\{\left(\mu_{j}, \phi_{j}\right)\right\}_{j}$ which are the eigenvalues and the eigenvectors of the matrix $\mathbf{S}$ such that

$$
\mathbf{S} \phi_{j}=\mu_{j} \phi_{j}
$$

Due to the symplectic nature of the matrix $\mathbf{S}$, its eigenvalues come in pairs as $\left(\mu_{j}, 1 / \mu_{j}\right)$. So the set $\left\{\left(\mu_{j}, \phi_{j}\right)\right\}_{j}$ can be partitioned into $n$ right-going wave modes $\left\{\left(\mu_{j}, \phi_{j}\right)\right\}_{j=1, \ldots, n}$ for which $\left|\mu_{j}\right|<1$, and $n$ left-going wave modes $\left\{\left(\mu_{j}^{\star}, \phi_{j}^{\star}\right)\right\}_{j=1, \ldots, n}$ for which $\mu_{j}^{\star}=1 / \mu_{j}$, with $\left|\mu_{j}^{\star}\right|>1$.

\subsection{Wave mode computation}

To find the eigenvectors and eigenvalues of $\mathbf{S}$, the so-called $\mathbf{S}+\mathbf{S}^{-1}$ transformation of the eigenproblem is considered [13], which yields an eigenproblem whose eigenvalues are of the form $\lambda_{j}=\mu_{j}+1 / \mu_{j}$, i.e.

$$
\left(\left(\mathbf{N}^{\prime} \mathbf{J} \mathbf{L}^{\prime T}+\mathbf{L}^{\prime} \mathbf{J} \mathbf{N}^{\prime} T\right)-\lambda_{j} \mathbf{L}^{\prime} \mathbf{J} \mathbf{L}^{\prime T}\right) \mathbf{z}_{j}=\mathbf{0}
$$

where

$$
\begin{gathered}
\mathbf{L}^{\prime}=\left[\begin{array}{cc}
\mathbf{0} & \mathbf{I}_{n} \\
\mathbf{D}_{\mathrm{LR}}^{*} & \mathbf{0}
\end{array}\right] \quad, \quad \mathbf{N}^{\prime}=\left[\begin{array}{cc}
\mathbf{D}_{\mathrm{RL}}^{*} & \mathbf{0} \\
-\left(\mathbf{D}_{\mathrm{LL}}^{*}+\mathbf{D}_{\mathrm{RR}}^{*}\right) & -\mathbf{I}_{n}
\end{array}\right] \quad, \quad \mathbf{J}=\left[\begin{array}{cc}
\mathbf{0} & \mathbf{I}_{n} \\
-\mathbf{I}_{n} & \mathbf{0}
\end{array}\right] . \\
\mathbf{N}^{\prime} \mathbf{J L}^{\prime T}+\mathbf{L}^{\prime} \mathbf{J} \mathbf{N}^{\prime}=\left[\begin{array}{cc}
\mathbf{D}_{\mathrm{RL}}^{*}-\mathbf{D}_{\mathrm{LR}}^{*} & \left(\mathbf{D}_{\mathrm{LL}}^{*}+\mathbf{D}_{\mathrm{RR}}^{*}\right) \\
-\left(\mathbf{D}_{\mathrm{LL}}^{*}+\mathbf{D}_{\mathrm{RR}}^{*}\right) & \mathbf{D}_{\mathrm{RL}}^{*}-\mathbf{D}_{\mathrm{LR}}^{*}
\end{array}\right],
\end{gathered}
$$

and

$$
\mathbf{L}^{\prime} \mathbf{J L}^{\prime T}=\mathbf{N}^{\prime} \mathbf{J N}^{\prime} T=\left[\begin{array}{cc}
\mathbf{0} & -\mathbf{D}_{\mathrm{RL}}^{*} \\
\mathbf{D}_{\mathrm{LR}}^{*} & \mathbf{0}
\end{array}\right] .
$$

One feature of the eigenproblem (6) is that it involves skew-symmetric matrices which are useful for speeding up the computation of the eigensolutions. Also, the eigenvectors $\left\{\mathbf{z}_{j}\right\}_{j}$ are expressed in terms of displacement/rotation components only. It is worth recalling that the eigenvalues of the eigenproblem come in pairs as $\left(\mu_{j}, \mu_{j}^{\star}=1 / \mu_{j}\right)$, meaning that the eigenvalues of the eigenproblem (6) are double, i.e., $\left(\lambda_{j}, \lambda_{j}^{\star}=\lambda_{j}\right)$. By considering the fact that $\lambda_{j}=$ $\mu_{j}+1 / \mu_{j}$, each pair of eigenvalues $\left(\mu_{j}, \mu_{j}^{\star}\right)$ can be found analytically by solving a quadratic equation of the form

$$
x^{2}-\lambda_{j} x+1=0,
$$

where it is understood that the relation $\mu_{j}^{\star}=1 / \mu_{j}$ can be verified with accurate precision. Also, there exist closed-form expressions linking the eigenvectors of the eigenproblem (6) to those of S (see [14]):

$$
\begin{gathered}
\mathbf{w}_{j}^{\prime}=\mathbf{J}\left(\mathbf{L}^{\prime T}-\mu_{j}^{\star} \mathbf{N}^{\prime T}\right) \mathbf{z}_{j} \quad, \quad \mathbf{w}_{j}^{\prime \star}=\mathbf{J}\left(\mathbf{L}^{\prime T}-\mu_{j} \mathbf{N}^{\prime T}\right) \mathbf{z}_{j} . \\
\boldsymbol{\phi}_{j}=\left[\begin{array}{cc}
\mathbf{I}_{n} & \mathbf{0} \\
\mathbf{D}_{\mathrm{RR}}^{*} & \mathbf{I}_{n}
\end{array}\right] \mathbf{w}_{j}^{\prime} \quad, \quad \boldsymbol{\phi}_{j}^{\star}=\left[\begin{array}{cc}
\mathbf{I}_{n} & \mathbf{0} \\
\mathbf{D}_{\mathrm{RR}}^{*} & \mathbf{I}_{n}
\end{array}\right] \mathbf{w}_{j}^{\prime \star},
\end{gathered}
$$

where $\left\{\mathbf{w}_{j}^{\prime}\right\}_{j}$ denote the eigenvectors associated to the eigenvalues $\left\{\mu_{j}^{\star}\right\}_{j}$ which are such that $\left|\mu_{j}^{\star}\right|>1$ and the wave shapes $\left\{\boldsymbol{\phi}_{j}\right\}_{j}$ and $\left\{\boldsymbol{\phi}_{j}^{\star}\right\}_{j}$ are the eigenvectors of $\mathbf{S}$. The state vector $\mathbf{u}_{\mathrm{L}}^{(k)}$ in section $k$ can be expanded as $\mathbf{u}_{\mathrm{L}}^{(k)}=\sum_{j} \exp \left(-i \beta_{j} d\right) Q_{j}^{(k-1)} \phi_{j}$. Also, the eigenvalues of $\mathbf{S}$ are expressed as $\mu_{j}=\exp \left(-i \beta_{j} d\right)$, where $\left\{\beta_{j}\right\}_{j}$ have the meaning of wavenumbers. 


\subsection{Forced response computation}

We consider here the structure shown in figure 2 which is made of a periodic part connected to two elastic subsystems. The focus is on a better modeling of the periodic part. In fact, there exist two main strategies for computing the forced response of a periodic structure, as shown in Figure 2, namely the dynamic stiffness matrix (DSM) approach and the wave amplitude (WA) approach. The DSM approach focuses on expressing a matrix relation to link the vector of forces/moments to that of displacements/rotations on the left and right ends of the structure. On the other hand, the WA approach focuses on assessing the wave reflection/transmission coefficients at the BCs while considering the spatial variation of the wave amplitudes along the structure.

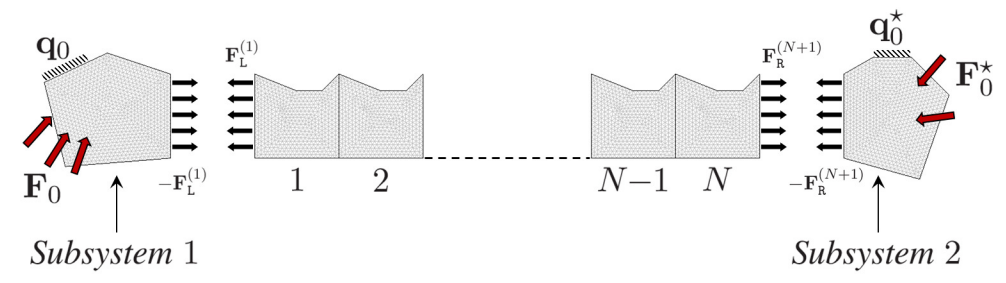

Figure 2: Periodic structure connected to two elastic subsystems.

\subsubsection{DSM approach}

The framework of the DSM approach is to consider the following matrix equation, which links the DOFs on the left and right ends of a periodic structure made up of $N$ substructures:

$$
\left[\begin{array}{c}
\mathbf{F}_{\mathrm{L}}^{(1)} \\
\mathbf{F}_{\mathrm{R}}^{(N+1)}
\end{array}\right]=\mathbf{D}_{s}\left[\begin{array}{c}
\mathbf{q}_{\mathrm{L}}^{(1)} \\
\mathbf{q}_{\mathrm{R}}^{(N+1)}
\end{array}\right],
$$

where the superscripts $(1)$ and $(N+1)$ denote the left and right ends of the structure, respectively. Also, $\mathbf{D}_{s}$ is the dynamic stiffness matrix of the structure which is condensed on its left and right ends. It is expressed as (see [3])

$$
\begin{aligned}
\mathbf{D}_{s}= & {\left[\begin{array}{cc}
\mathbf{D}_{\mathrm{LL}}^{*} & \mathbf{0} \\
\mathbf{0} & \mathbf{D}_{\mathrm{RR}}^{*}
\end{array}\right]+\left[\begin{array}{cc}
\mathbf{D}_{\mathrm{LR}}^{*} & \mathbf{0} \\
\mathbf{0} & \mathbf{D}_{\mathrm{RL}}^{*}
\end{array}\right]\left[\begin{array}{cc}
\boldsymbol{\Phi}_{\mathrm{q}}^{\star} \boldsymbol{\mu}^{N-1} \boldsymbol{\Phi}_{\mathrm{q}}^{\star-1} & \boldsymbol{\Phi}_{\mathrm{q}} \boldsymbol{\mu} \boldsymbol{\Phi}_{\mathrm{q}}^{-1} \\
\boldsymbol{\Phi}_{\mathrm{q}}^{\star} \boldsymbol{\mu} \boldsymbol{\Phi}_{\mathrm{q}}^{\star-1} & \boldsymbol{\Phi}_{\mathrm{q}} \boldsymbol{\mu}^{N-1} \boldsymbol{\Phi}_{\mathrm{q}}^{-1}
\end{array}\right] } \\
& \times\left[\begin{array}{cc}
\boldsymbol{\Phi}_{\mathrm{q}}^{\star} \boldsymbol{\mu}^{N} \boldsymbol{\Phi}_{\mathrm{q}}^{\star-1} & \mathbf{I}_{n} \\
\mathbf{I}_{n} & \boldsymbol{\Phi}_{\mathrm{q}} \boldsymbol{\mu}^{N} \boldsymbol{\Phi}_{\mathrm{q}}^{-1}
\end{array}\right]
\end{aligned}
$$

where $\Phi_{\mathrm{q}}$ and $\Phi_{\mathrm{q}}^{\star}$ are square $n \times n$ matrices defined as $\Phi_{\mathrm{q}}=\left[\phi_{\mathrm{q} 1} \cdots \phi_{\mathrm{q} n}\right]$ and $\Phi_{\mathrm{q}}^{\star}=\left[\boldsymbol{\phi}_{\mathrm{q} 1}^{\star} \cdots \phi_{\mathrm{q} n}^{\star}\right]$. Also, $\boldsymbol{\mu}$ is a $n \times n$ diagonal matrix whose components relate the eigenvalues for the right-going wave modes (see Section 2.1), i.e., $\boldsymbol{\mu}=\operatorname{diag}\left\{\mu_{j}\right\}_{j=1, \ldots, n}$. Notice that the 2 -norm [10] of the matrix $\boldsymbol{\mu}$ is less than one, i.e., $\|\boldsymbol{\mu}\|_{2}<1$, which is explained because $\|\boldsymbol{\mu}\|_{2}=\max \left|\mu_{j}\right|<1$ by convention. The dynamic stiffness matrix $\mathbf{D}_{s}$ of the periodic structure can be readily assembled with the dynamic stiffness matrices of other elastic subsystems as this is usually done in the FE method, with a view to expressing a global dynamic stiffness matrix. 


\subsubsection{WA approach}

Within the WFE framework, the vectors of displacements/rotations and forces/moments on the substructure boundary $(k)$ are estimated by means of a wave expansion, as follows [8]:

$$
\mathbf{q}^{(k)}=\boldsymbol{\Phi}_{\mathrm{q}} \boldsymbol{\mu}^{k-1} \mathbf{Q}+\boldsymbol{\Phi}_{\mathrm{q}}^{\star} \boldsymbol{\mu}^{N+1-k} \mathbf{Q}^{\star}, \quad \pm \mathbf{F}^{(k)}=\boldsymbol{\Phi}_{\mathrm{F}} \boldsymbol{\mu}^{k-1} \mathbf{Q}+\boldsymbol{\Phi}_{\mathrm{F}}^{\star} \boldsymbol{\mu}^{N+1-k} \mathbf{Q}^{\star},
$$

where $\Phi_{\mathrm{F}}=\left[\phi_{\mathrm{F} 1} \cdots \phi_{\mathrm{F} n}\right]$ and $\Phi_{\mathrm{F}}^{\star}=\left[\phi_{\mathrm{F} 1}^{\star} \cdots \phi_{\mathrm{F} n}^{\star}\right]$. Also, in Eq. (15), $\mathrm{Q}$ and $\mathrm{Q}^{\star}$ are vectors of wave amplitudes at the left and right ends of the whole periodic structure, respectively. Finally, the sign ahead of $\mathbf{F}$ results from the choice of description considered, i.e., it is negative when the left boundary of a substructure is of concern, and positive for the right boundary.

The determination of the vectors of wave amplitudes $\mathrm{Q}$ and $\mathrm{Q}^{\star}$ involves considering the $\mathrm{BCs}$ on the left and right ends of the whole periodic structure. For instance, regarding the case shown in Figure 2 when the left and right ends of the periodic structure are connected to extra elastic subsystems, the BCs are expressed as:

$$
-\mathbf{F}_{\mathrm{L}}^{(1)}=\mathbb{D} \mathbf{q}_{\mathrm{L}}^{(1)}+\mathbb{D}_{\mathrm{q}} \mathbf{q}_{0}+\mathbb{D}_{\mathrm{F}} \mathbf{F}_{0} \quad, \quad-\mathbf{F}_{\mathrm{R}}^{(N+1)}=\mathbb{D}^{\star} \mathbf{q}_{\mathrm{R}}^{(N+1)}+\mathbb{D}_{\mathbf{q}}^{\star} \mathbf{q}_{0}^{\star}+\mathbb{D}_{\mathrm{F}}^{\star} \mathbf{F}_{0}^{\star},
$$

where $\mathbb{D}$ and $\mathbb{D}^{\star}$ denote the dynamic stiffness matrices of the subsystems which are condensed on the interface DOFs. Also, $\mathbb{D}_{\mathrm{q}}$ and $\mathbb{D}_{\mathrm{q}}^{\star}$ (resp. $\mathbb{D}_{\mathrm{F}}$ and $\mathbb{D}_{\mathrm{F}}^{\star}$ ) are matrices which reflect the effects of prescribed vectors of displacements/rotations $\left(\mathbf{q}_{0}, \mathbf{q}_{0}^{\star}\right)$ (resp. prescribed vectors of forces/moments $\left.\left(\mathbf{F}_{0}, \mathbf{F}_{0}^{\star}\right)\right)$ that may be applied to the subsystems.

By considering the wave expansion (15), the BCs can be expressed in wave-based form, as follows:

$$
\mathbf{Q}=\mathbb{C} \boldsymbol{\mu}^{N} \mathbf{Q}^{\star}+\mathbb{F} \quad, \quad \mathbf{Q}^{\star}=\mathbb{C}^{\star} \boldsymbol{\mu}^{N} \mathbf{Q}+\mathbb{F}^{\star},
$$

where $\mathbb{C}$ and $\mathbb{C}^{\star}$ are $n \times n$ scattering matrices whose components relate the reflection coefficients for the wave modes incident to the boundaries, while $\mathbb{F}$ and $\mathbb{F}^{\star}$ are $n \times 1$ vectors which relate the effects of the excitation sources. Expressions for $\mathbb{C}, \mathbb{C}^{\star}, \mathbb{F}$ and $\mathbb{F}^{\star}$ follow from the aforementioned BCs, i.e:

$$
\begin{array}{lll}
\mathbb{C}=-\left[\mathbb{D} \boldsymbol{\Phi}_{\mathrm{q}}-\boldsymbol{\Phi}_{\mathrm{F}}\right]^{-1}\left[\mathbb{D} \boldsymbol{\Phi}_{\mathrm{q}}^{\star}-\boldsymbol{\Phi}_{\mathrm{F}}^{\star}\right] & , \quad \mathbb{C}^{\star}=-\left[\mathbb{D}^{\star} \boldsymbol{\Phi}_{\mathrm{q}}^{\star}+\boldsymbol{\Phi}_{\mathrm{F}}^{\star}\right]^{-1}\left[\mathbb{D}^{\star} \boldsymbol{\Phi}_{\mathrm{q}}+\boldsymbol{\Phi}_{\mathrm{F}}\right] \\
\mathbb{F}=-\left[\mathbb{D} \boldsymbol{\Phi}_{\mathrm{q}}-\boldsymbol{\Phi}_{\mathrm{F}}\right]^{-1}\left[\mathbb{D}_{\mathrm{q}} \mathbf{q}_{0}+\mathbb{D}_{\mathrm{F}} \mathbf{F}_{0}\right] \quad, \quad \mathbb{F}^{\star}=-\left[\mathbb{D}^{\star} \boldsymbol{\Phi}_{\mathrm{q}}+\boldsymbol{\Phi}_{\mathrm{F}}\right]^{-1}\left[\mathbb{D}_{\mathrm{q}}^{\star} \mathbf{q}_{0}^{\star}+\mathbb{D}_{\mathrm{F}}^{\star} \mathbf{F}_{0}^{\star}\right] .
\end{array}
$$

By considering the wave-based BCs, Eq. (17), a whole matrix equation is established as follows:

$$
\mathcal{A Q}=\mathcal{F}
$$

where

$$
\mathcal{A}=\left[\begin{array}{cc}
\mathbf{I}_{n} & -\mathbb{C} \boldsymbol{\mu}^{N} \\
-\mathbb{C}^{\star} \boldsymbol{\mu}^{N} & \mathbf{I}_{n}
\end{array}\right] \quad, \quad \mathcal{Q}=\left[\begin{array}{c}
\mathrm{Q} \\
\mathbf{Q}^{\star}
\end{array}\right] \quad, \quad \mathcal{F}=\left[\begin{array}{c}
\mathbb{F} \\
\mathbb{F}^{\star}
\end{array}\right] .
$$

Solving the matrix equation (19) yields the vectors of wave amplitudes as $\mathcal{Q}=\mathcal{A}^{-1} \mathcal{F}$.

\section{Model reduction}

\subsection{Motivation and framework}

As it is well known, waves traveling along a structure like the one shown in Figure 1 can be classified into weakly evanescent, which would be propagative if there were no damping, and strongly evanescent, i.e., which decay exponentially from the excitation sources. So, one may assume that far from the sources these evanescent waves are weakly contributing to the 
structure dynamics. Here, a central periodic structure consisting of $N-2$ substructures is considered which is surrounded by two extra substructures. Such a central periodic structure is analyzed by means of the WFE method (Section 2.3), while the extra substructures are modeled by means of the FE method and are treated as BCs like those involved by Eq. (18).

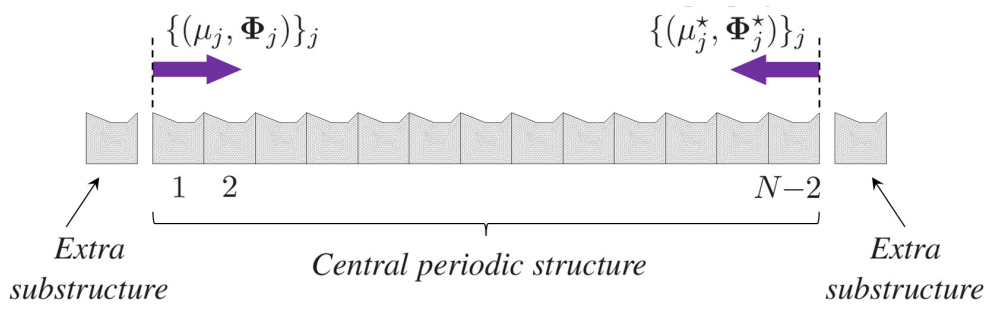

Figure 3: Central periodic structure surrounded by two extra substructures.

Prior to any rigorous derivation, a simple qualitative analysis is proposed here to quantify the error made when some strong evanescent wave modes are neglected in the WFE-based formulations. Consider the central periodic structure as shown in Figure 3, which is enclosed between the substructure boundary (1) and the substructure boundary $(N-1)$. Assume that the excitation sources occur on the left boundary of the left extra substructure, and the right boundary of the right extra substructure, only. Denote as $\mathrm{Q}$ the vector of wave amplitudes at the left end of the left extra substructure, and $Q^{\star}$ the vector of wave amplitudes at the right end of the right extra substructure. By considering the wave expansion (15), the vectors of displacements/rotations and forces/moments along the central periodic structure are expressed as

$$
\begin{aligned}
\mathbf{q}^{(k)} & =\sum_{j=1}^{n}\left(\mu_{j}^{k} \mathbf{q}_{j}+\mu_{j}^{N-k} \mathbf{q}_{j}^{\star}\right)=\sum_{j=1}^{n} \mu_{j}\left(\mu_{j}^{k-1} \mathbf{q}_{j}+\mu_{j}^{N-1-k} \mathbf{q}_{j}^{\star}\right), \\
\pm \mathbf{F}^{(k)} & =\sum_{j=1}^{n}\left(\mu_{j}^{k} \mathbf{F}_{j}+\mu_{j}^{N-k} \mathbf{F}_{j}^{\star}\right)=\sum_{j=1}^{n} \mu_{j}\left(\mu_{j}^{k-1} \mathbf{F}_{j}+\mu_{j}^{N-1-k} \mathbf{F}_{j}^{\star}\right),
\end{aligned}
$$

where $\mathbf{q}_{j}=Q_{j} \boldsymbol{\Phi}_{\mathrm{q} j}, \mathbf{q}_{j}^{\star}=Q_{j}^{\star} \boldsymbol{\Phi}_{\mathrm{q} j}, \mathbf{F}_{j}=Q_{j} \boldsymbol{\Phi}_{\mathrm{F} j}$ and $\mathbf{F}_{j}^{\star}=Q_{j}^{\star} \boldsymbol{\Phi}_{\mathrm{F} j}^{\star}$. Since the integer $k$ is such that $1 \leq k \leq N-1$, one has $k-1 \geq 0$ and $N-1-k \geq 0$, and then $\left|\mu_{j}\right|^{k-1} \leq 1$ and $\left|\mu_{j}\right|^{N-1-k} \leq 1$. This is explained because $\left|\mu_{j}\right|<1$ by convention. Let us denote as $\overline{\mathbf{q}}^{(k)}$ and $\overline{\mathbf{F}}^{(k)}$ the parts of the vectors of displacements/rotations and forces/moments which are induced by strongly evanescent wave modes, only. Denote as $\left\{\left(\bar{\mu}_{j}, \overline{\boldsymbol{\phi}}_{j}\right)\right\}_{j=1, \ldots, n-m}$ and $\left\{\left(\bar{\mu}_{j}^{\star}, \bar{\phi}_{j}^{\star}\right)\right\}_{j=1, \ldots, n-m}$ those evanescent wave modes which are such that $\left\{\left(\bar{\mu}_{j}, \bar{\phi}_{j}\right)\right\}_{j=1, \ldots, n-m} \subset\left\{\left(\mu_{j}, \phi_{j}\right)\right\}_{j=1, \ldots, n}$ and $\left\{\left(\bar{\mu}_{j}^{\star}, \overline{\boldsymbol{\phi}}_{j}^{\star}\right)\right\}_{j=1, \ldots, n-m} \subset\left\{\left(\mu_{j}^{\star}, \boldsymbol{\phi}_{j}^{\star}\right)\right\}_{j=1, \ldots, n}$ where $m>1$. From Eqs. (21) and (22), $\overline{\mathbf{q}}^{(k)}$ and $\overline{\mathbf{F}}^{(k)}$ are expressed as

$$
\begin{gathered}
\left\|\overline{\mathbf{q}}^{(k)}\right\|=\left\|\sum_{j=1}^{n-m} \bar{\mu}_{j}\left(\bar{\mu}_{j}^{k-1} \overline{\mathbf{q}}_{j}+\bar{\mu}_{j}^{N-1-k} \overline{\mathbf{q}}_{j}^{\star}\right)\right\| \leq \sum_{j=1}^{n-m}\left|\bar{\mu}_{j}\right|\left(\left\|\overline{\mathbf{q}}_{j}\right\|+\left\|\overline{\mathbf{q}}_{j}^{\star}\right\|\right), \\
\left\|\overline{\mathbf{F}}^{(k)}\right\|=\left\|\sum_{j=1}^{n-m} \bar{\mu}_{j}\left(\bar{\mu}_{j}^{k-1} \overline{\mathbf{F}}_{j}+\bar{\mu}_{j}^{N-1-k} \overline{\mathbf{F}}_{j}^{\star}\right)\right\| \leq \sum_{j=1}^{n-m}\left|\bar{\mu}_{j}\right|\left(\left\|\overline{\mathbf{F}}_{j}\right\|+\left\|\overline{\mathbf{F}}_{j}^{\star}\right\|\right),
\end{gathered}
$$

where $\|$.$\| denotes the 2$-norm. Since highly evanescent wave modes are of concern, one expects $\left|\bar{\mu}_{j}\right| \ll 1$. To summarize, it seems logical to neglect these wave modes. 


\subsection{Reduced modelings}

\subsubsection{DSM approach}

By expanding the vectors of displacements/rotations and forces/moments of the structure onto the reduced wave basis $\left\{\tilde{\boldsymbol{\phi}}_{j}\right\}_{j=1, \ldots, m} \cup\left\{\tilde{\boldsymbol{\phi}}_{j}^{\star}\right\}_{j=1, \ldots, m}$, the dynamic stiffness matrix of the central periodic structure - namely, $\mathbf{D}_{s}^{r}$ - can be approximated as $\mathbf{D}_{s}^{r} \approx \tilde{\mathbf{D}}_{s}^{r}$, where $\tilde{\mathbf{D}}_{s}^{r}$ is defined so that

$$
\left[\begin{array}{c}
\tilde{\mathbf{F}}_{\mathrm{L}}^{(1)} \\
\tilde{\mathbf{F}}_{\mathrm{R}}^{(N-1)}
\end{array}\right]=\tilde{\mathbf{D}}_{s}^{r}\left[\begin{array}{c}
\tilde{\mathbf{q}}_{\mathrm{L}}^{(1)} \\
\tilde{\mathbf{q}}_{\mathrm{R}}^{(N-1)}
\end{array}\right] .
$$

Here, $\tilde{\mathbf{q}}_{\mathrm{L}}^{(1)}$ and $\tilde{\mathbf{F}}_{\mathrm{L}}^{(1)}$ (resp. $\tilde{\mathbf{q}}_{\mathrm{R}}^{(N-1)}$ and $\tilde{\mathbf{F}}_{\mathrm{R}}^{(N-1)}$ ) refer to the approximated vectors of displacements/rotations and forces/vectors, respectively, on the left (resp. right) end of the central structure. The derivation of the dynamic stiffness matrix $\tilde{\mathbf{D}}_{s}^{r}$ follows from that of $\mathbf{D}_{s}$, i.e.

$$
\begin{aligned}
\tilde{\mathbf{D}}_{s}^{r}= & {\left[\begin{array}{cc}
\mathbf{D}_{\mathrm{LL}}^{*} & \mathbf{0} \\
\mathbf{0} & \mathbf{D}_{\mathrm{RR}}^{*}
\end{array}\right]+\left[\begin{array}{cc}
\mathbf{D}_{\mathrm{LR}}^{*} & \mathbf{0} \\
\mathbf{0} & \mathbf{D}_{\mathrm{RL}}^{*}
\end{array}\right]\left[\begin{array}{cc}
\tilde{\boldsymbol{\Phi}}_{\mathrm{q}}^{\star} \tilde{\boldsymbol{\mu}}^{N-3} \tilde{\boldsymbol{\Phi}}_{\mathrm{q}}^{\star+} & \tilde{\boldsymbol{\Phi}}_{\mathrm{q}} \tilde{\boldsymbol{\mu}} \tilde{\boldsymbol{\Phi}}_{\mathrm{q}}^{+} \\
\boldsymbol{\Phi}_{\mathrm{q}}^{\star} \tilde{\boldsymbol{\mu}} \tilde{\boldsymbol{\Phi}}_{\mathrm{q}}^{\star+} & \tilde{\boldsymbol{\Phi}}_{\mathrm{q}} \tilde{\boldsymbol{\mu}}^{N-3} \boldsymbol{\Phi}_{\mathrm{q}}^{+}
\end{array}\right] } \\
& \times\left[\begin{array}{cc}
\tilde{\boldsymbol{\Phi}}_{\mathrm{q}}^{\star} \tilde{\boldsymbol{\mu}}^{N-2} \tilde{\boldsymbol{\Phi}}_{\mathrm{q}}^{\star+} & \mathbf{I}_{m} \\
\mathbf{I}_{m} & \tilde{\boldsymbol{\Phi}}_{\mathrm{q}} \tilde{\boldsymbol{\mu}}^{N-2} \tilde{\boldsymbol{\Phi}}_{\mathrm{q}}^{+}
\end{array}\right]^{-1}
\end{aligned}
$$

where $\tilde{\boldsymbol{\Phi}}_{\mathrm{q}}$ and $\tilde{\boldsymbol{\Phi}}_{\mathrm{q}}^{\star}$ are $n \times m$ rectangular matrices defined so that $\tilde{\boldsymbol{\Phi}}_{\mathrm{q}}=\left[\tilde{\boldsymbol{\phi}}_{\mathrm{q} 1} \cdots \tilde{\boldsymbol{\phi}}_{\mathrm{q} m}\right]$ and $\tilde{\boldsymbol{\Phi}}_{\mathrm{q}}^{\star}=\left[\tilde{\boldsymbol{\phi}}_{\mathrm{q} 1}^{\star} \cdots \tilde{\phi}_{\mathrm{q} m}^{\star}\right]$; also $\tilde{\boldsymbol{\mu}}$ is a $m \times m$ diagonal matrix defined as $\tilde{\boldsymbol{\mu}}=\operatorname{diag}\left\{\tilde{\mu}_{j}\right\}_{j=1, \ldots, m}$; finally, $\tilde{\boldsymbol{\Phi}}_{\mathrm{q}}^{+}$and $\tilde{\boldsymbol{\Phi}}_{\mathrm{q}}^{\star+}$ are the left pseudo-inverses of $\tilde{\boldsymbol{\Phi}}_{\mathrm{q}}$ and $\tilde{\boldsymbol{\Phi}}_{\mathrm{q}}^{\star}$, which are defined so that $\tilde{\boldsymbol{\Phi}}_{\mathrm{q}}^{+}=\left[\tilde{\boldsymbol{\Phi}}_{\mathrm{q}}^{H} \tilde{\boldsymbol{\Phi}}_{\mathrm{q}}\right]^{-1} \tilde{\boldsymbol{\Phi}}_{\mathrm{q}}^{H}$ and $\tilde{\boldsymbol{\Phi}}_{\mathrm{q}}^{\star+}=\left[\tilde{\boldsymbol{\Phi}}_{\mathrm{q}}^{\star H} \tilde{\boldsymbol{\Phi}}_{\mathrm{q}}^{\star}\right]^{-1} \tilde{\boldsymbol{\Phi}}_{\mathrm{q}}^{\star H}$, where the superscript $H$ is the conjugate transpose. The dynamic stiffness matrix $\tilde{\mathbf{D}}_{s}^{r}$ is to be assembled with the dynamic stiffness matrices of the left and right extra substructures (Figure 3) to express the dynamic stiffness matrix $\tilde{\mathbf{D}}_{s}$ of the whole periodic structure, i.e., the one composed of $N$ substructures.

\subsubsection{WA approach}

From Eq. (15), the approximated vectors of nodal displacements/rotations and forces/moments on a substructure boundary $(k)(1 \leq k \leq N-1)$ along the central periodic structure are expressed as

$$
\tilde{\mathbf{q}}^{(k)}=\tilde{\boldsymbol{\Phi}}_{\mathrm{q}} \tilde{\boldsymbol{\mu}}^{k-1} \tilde{\mathbf{Q}}+\tilde{\boldsymbol{\Phi}}_{\mathrm{q}}^{\star} \tilde{\boldsymbol{\mu}}^{N-1-k} \tilde{\mathbf{Q}}^{\star} \quad, \quad \pm \tilde{\mathbf{F}}^{(k)}=\tilde{\boldsymbol{\Phi}}_{\mathrm{F}} \tilde{\boldsymbol{\mu}}^{k-1} \tilde{\mathbf{Q}}+\tilde{\boldsymbol{\Phi}}_{\mathrm{F}}^{\star} \tilde{\boldsymbol{\mu}}^{N-1-k} \tilde{\mathbf{Q}}^{\star}
$$

where $\tilde{\boldsymbol{\Phi}}_{\mathrm{q}}=\left[\tilde{\boldsymbol{\phi}}_{\mathrm{q} 1} \cdots \tilde{\boldsymbol{\phi}}_{\mathrm{q} m}\right], \tilde{\boldsymbol{\Phi}}_{\mathrm{q}}^{\star}=\left[\tilde{\boldsymbol{\phi}}_{\mathrm{q} 1}^{\star} \cdots \tilde{\boldsymbol{\phi}}_{\mathrm{q} m}^{\star}\right], \tilde{\boldsymbol{\Phi}}_{\mathrm{F}}=\left[\tilde{\boldsymbol{\phi}}_{\mathrm{F} 1} \cdots \tilde{\boldsymbol{\phi}}_{\mathrm{F} m}\right]$ and $\tilde{\boldsymbol{\Phi}}_{\mathrm{F}}^{\star}=\left[\tilde{\boldsymbol{\phi}}_{\mathrm{F} 1}^{\star} \cdots \tilde{\boldsymbol{\phi}}_{\mathrm{F} m}^{\star}\right]$. The computation of $\tilde{\mathbf{Q}}$ and $\tilde{\mathrm{Q}}^{\star}$ follows from the following reduced matrix equation (cf. Eq. (19)):

$$
\tilde{\mathcal{A}} \tilde{\mathcal{Q}}=\tilde{\mathcal{F}},
$$

where

$$
\begin{array}{r}
\tilde{\mathcal{A}}=\left[\begin{array}{cc}
\mathbf{I}_{m} & -\tilde{\mathbb{C}} \tilde{\boldsymbol{\mu}}^{N-2} \\
-\tilde{\mathbb{C}}^{\star} \tilde{\boldsymbol{\mu}}^{N-2} & \mathbf{I}_{m}
\end{array}\right] \quad, \quad \tilde{\mathcal{Q}}=\left[\begin{array}{c}
\tilde{\mathbf{Q}} \\
\tilde{\mathbf{Q}}^{\star}
\end{array}\right] \quad, \quad \tilde{\mathcal{F}}=\left[\begin{array}{c}
\tilde{\mathbb{F}} \\
\tilde{\mathbb{F}}^{\star}
\end{array}\right] . \\
\tilde{\mathbb{C}}=-\left[\mathbb{D} \tilde{\boldsymbol{\Phi}}_{\mathrm{q}}-\tilde{\boldsymbol{\Phi}}_{\mathrm{F}}\right]^{+}\left[\mathbb{D} \tilde{\boldsymbol{\Phi}}_{\mathrm{q}}^{\star}-\tilde{\boldsymbol{\Phi}}_{\mathrm{F}}^{\star}\right], \quad \tilde{\mathbb{C}}^{\star}=-\left[\mathbb{D}^{\star} \tilde{\boldsymbol{\Phi}}_{\mathrm{q}}^{\star}+\tilde{\boldsymbol{\Phi}}_{\mathrm{F}}^{\star}\right]^{+}\left[\mathbb{D}^{\star} \tilde{\boldsymbol{\Phi}}_{\mathrm{q}}+\tilde{\boldsymbol{\Phi}}_{\mathrm{F}}\right], \\
\tilde{\mathbb{F}}=-\left[\mathbb{D} \tilde{\boldsymbol{\Phi}}_{\mathrm{q}}-\tilde{\boldsymbol{\Phi}}_{\mathrm{F}}\right]^{+}\left[\mathbb{D}_{\mathrm{q}} \mathbf{q}_{0}+\mathbb{D}_{\mathrm{F}} \mathbf{F}_{0}\right] \quad, \quad \tilde{\mathbb{F}}^{\star}=-\left[\mathbb{D}^{\star} \tilde{\boldsymbol{\Phi}}_{\mathrm{q}}+\tilde{\boldsymbol{\Phi}}_{\mathrm{F}}\right]^{+}\left[\mathbb{D}_{\mathrm{q}}^{\star} \mathbf{q}_{0}^{\star}+\mathbb{D}_{\mathrm{F}}^{\star} \mathbf{F}_{0}^{\star}\right],
\end{array}
$$

where the superscript + denotes the left pseudo-inverse. 


\subsection{Error indicator}

The selection of the number $m$ of right-going and left-going wave modes in the central periodic structure that need to be computed from the Lanczos method is addressed here using the strategy proposed in [11]. In this work, it is suggested to bound the relative errors, for the vectors of nodal displacements/rotations and forces/moments, as follows:

$$
\frac{\left\|\widetilde{\mathbf{q}}^{(k)}-\mathbf{q}^{(k)}\right\|}{\left\|\mathbf{q}^{(k)}\right\|} \leq \mathcal{E}_{s} \quad, \quad \frac{\left\|\widetilde{\mathbf{F}}^{(k)}-\mathbf{F}^{(k)}\right\|}{\left\|\mathbf{F}^{(k)}\right\|} \leq \mathcal{E}_{s} \quad \forall k,
$$

where

$$
\begin{aligned}
\mathcal{E}_{s}=\max \left\{\left[\left(\epsilon_{1}^{\mathbf{E}}+\epsilon_{2}^{\mathbf{E}}\right)+\frac{\left\|\mathbf{A}^{s}\right\|}{1-\left\|\mathbf{A}^{s}\right\|}\left(\epsilon_{1}^{\mathbf{A}}+\epsilon_{2}^{\mathbf{A}}\right)\right] \frac{1+\left\|\mathbf{A}^{s}\right\|}{1-\left\|\mathbf{A}^{s}\right\|},\right. \\
\\
{\left.\left[\left(\epsilon_{1}^{\mathbf{E}^{\star}}+\epsilon_{2}^{\mathbf{E}^{\star}}\right)+\frac{\left\|\mathbf{A}^{\star s}\right\|}{1-\left\|\mathbf{A}^{\star s}\right\|}\left(\epsilon_{1}^{\mathbf{A}^{\star}}+\epsilon_{2}^{\mathbf{A}^{\star}}\right)\right] \frac{1+\left\|\mathbf{A}^{\star s}\right\|}{1-\left\|\mathbf{A}^{\star s}\right\|}\right\} . }
\end{aligned}
$$

Within the present framework, those matrix/vector terms are expressed as:

$$
\begin{array}{ll}
\mathbf{A}=\mathbb{C} \boldsymbol{\mu}^{N-2} \mathbb{C}^{\star} \boldsymbol{\mu}^{N-2}, & \mathbf{A}^{\star}=\mathbb{C}^{\star} \boldsymbol{\mu}^{N-2} \mathbb{C}^{\boldsymbol{\mu}^{N-2},}, \quad \mathbf{B}=\mathbb{C} \boldsymbol{\mu}^{N-2} \mathbb{F}^{\star}+\mathbb{F} \\
\mathbf{B}^{\star}=\mathbb{C}^{\star} \boldsymbol{\mu}^{N-2} \mathbb{F}+\mathbb{F}^{\star}, & \quad \mathbf{E}_{s}=\left(\sum_{p=0}^{s-1} \mathbf{A}^{p}\right) \mathbf{B}, \quad \mathbf{E}_{s}^{\star}=\left(\sum_{p=0}^{s-1} \mathbf{A}^{\star p}\right) \mathbf{B}^{\star},
\end{array}
$$

where $(\mathbb{C}, \mathbb{F})$ and $\left(\mathbb{F}^{\star}, \mathbb{C}^{\star}\right)$ are scattering matrices and vectors of excitation sources that relate the coupling conditions between the central structure and the extra substructures, see Eqs. (18). Also, in Eq. (34), $s$ is an integer defined so that

$$
s=\max \left\{u:\left\|\mathbf{A}^{u}\right\| \geq 0.1 \text { and }\left\|\mathbf{A}^{\star u}\right\| \geq 0.1\right\} .
$$

Besides, in Eq. (33), $\epsilon_{1}^{\mathbf{E}}, \epsilon_{2}^{\mathbf{E}}, \epsilon_{1}^{\mathbf{E}^{\star}}$ and $\epsilon_{2}^{\mathbf{E}^{\star}}$ are error parameters expressed as

$$
\begin{gathered}
\epsilon_{1}^{\mathbf{E}}=\frac{\| \widetilde{\mathbf{E}_{s}-\widetilde{\mathcal{L}} \mathbf{E}_{s} \|}}{\left\|\mathbf{E}_{s}\right\|}, \quad \epsilon_{2}^{\mathbf{E}}=\frac{\left\|\mathcal{L}_{\mathrm{r}} \mathbf{E}_{s}\right\|}{\left\|\mathbf{E}_{s}\right\|}, \quad \epsilon_{1}^{\mathbf{E}^{\star}}=\frac{\left\|\widetilde{\mathbf{E}}_{s}^{\star}-\widetilde{\mathcal{L}} \mathbf{E}_{s}^{\star}\right\|}{\left\|\mathbf{E}_{s}^{\star}\right\|}, \quad \epsilon_{2}^{\mathbf{E}^{\star}}=\frac{\left\|\mathcal{L}_{\mathrm{r}} \mathbf{E}_{s}^{\star}\right\|}{\left\|\mathbf{E}_{s}^{\star}\right\|}, \\
\epsilon_{1}^{\mathbf{A}}=\frac{\left\|\widetilde{\mathbf{A}^{s}} \widetilde{\mathcal{L}}-\widetilde{\mathcal{L}} \mathbf{A}^{s}\right\|}{\left\|\mathbf{A}^{s}\right\|}, \quad \epsilon_{2}^{\mathbf{A}}=\frac{\left\|\mathcal{L}_{\mathrm{r}} \mathbf{A}^{s} \widetilde{\mathcal{L}}^{T}\right\|}{\left\|\mathbf{A}^{s}\right\|}, \quad \epsilon_{1}^{\mathbf{A}^{\star}}=\frac{\left\|\widetilde{\mathbf{A}}{ }^{\star s} \widetilde{\mathcal{L}}-\widetilde{\mathcal{L}} \mathbf{A}^{\star s}\right\|}{\left\|\mathbf{A}^{\star s}\right\|}, \quad \epsilon_{2}^{\mathbf{A}^{\star}}=\frac{\left\|\mathcal{L}_{\mathrm{r}} \mathbf{A}^{\star s} \widetilde{\mathcal{L}}^{T}\right\|}{\left\|\mathbf{A}^{\star s}\right\|},
\end{gathered}
$$

where $\mathcal{L}_{\mathrm{r}}$ and $\widetilde{\mathcal{L}}$ are Boolean incidence matrices that localize, respectively, the rejected and retained wave modes. One of the features of the proposed error analysis is that the error bound $\mathcal{E}_{s}$ is to be computed at the maximum discrete frequency considered within the frequency band of interest, only, as explained in [11].

\section{Numerical experiments}

To highlight the efficiency of the proposed reduction method (Section 3), two test cases are investigated that concern: (i) a 2D periodic structure undergoing plane stresses (see Figure 1); (ii) a 3D cylindrical shell with a periodic distribution of longitudinal and circumferential stiffeners (see Figure 7). Both structures exhibit substructures which are not symmetric. Within the framework of the DSM and WA approaches, the FRFs of the structures are computed using MATLAB ${ }^{\circledR}$. These are compared with reference results issued from the conventional FE method when the FRFs are computed using a commercial FE software. 


\subsection{D periodic structure}

As a first example, the frequency forced response of a 2D periodic structure, undergoing plane stresses, is investigated (see Figure 1). The whole periodic structure is made up of $N=15$ identical substructures whose dimensions are shown in Figure 4. Each substructure is meshed by means of $2 \mathrm{D}$ linear triangular elements whose nodes exhibit horizontal and vertical displacement DOFs. Also, the structure exhibits the following material characteristics: Young's modulus of $210 \mathrm{GPa}$, density of $7800 \mathrm{~kg} / \mathrm{m}^{3}$, Poisson's ratio of 0.3 and a loss factor of 0.001 . Also, its thickness is $0.001 \mathrm{~m}$. The structure is excited by a unit point force acting along the horizontal $x$-direction at the bottom corner of the left end; also, it is clamped over its right end.

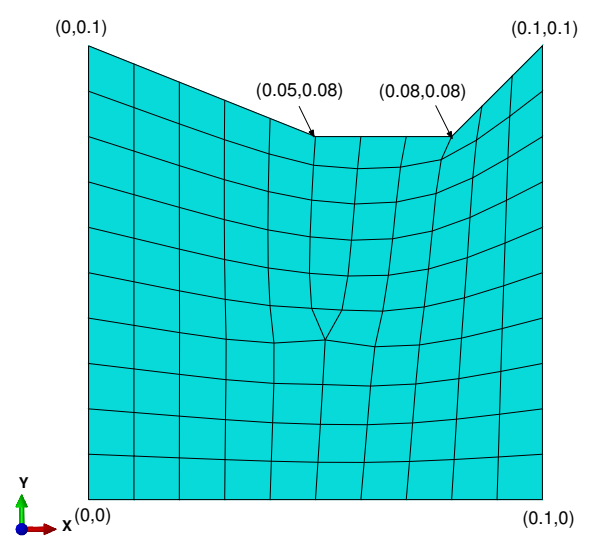

Figure 4: Geometry and mesh of a substructure composing the 2D periodic structure displayed in Figure 1 (dimensions are provided in meters).

The DSM approach proposed in Section 3.2.1 is used to compute the FRF of the structure i.e., the frequency evolution of the modulus of the displacement at the location of the input force — over a frequency band $[1 \mathrm{~Hz}, 2000 \mathrm{~Hz}]$. For this purpose, the reduction method proposed in Section 3 is investigated. It consists in dividing the whole periodic structure into one central structure having $N-2=13$ substructures, which is surrounded by two extra-substructures (Figure 3). The FRF of the whole structure is computed by considering different numbers $m$ of right-going and left-going wave modes. The related results are displayed in Figure 5 along with the reference FE solution, i.e., which is issued from a commercial FE software. Here, a moderately dense mesh is used, leading to $n=22$ DOFs over the left/right boundary of each substructure. As it can be seen, considering $m=2$ right/left-going wave modes of the central structure is clearly not enough. However, considering three or more wave modes (i.e., $m \geq 3$ ) yields the expected results with accurate precision. This might be explained by investigating the first four propagation constants (see Table 1) - namely, $\mu_{1}, \mu_{2}, \mu_{3}$ and $\mu_{4}$ - at $2000 \mathrm{~Hz}$, among all the 22 propagation constants whose moduli are less than one. Here, it appears that two waves are slowly damped, one is moderately decreasing while the last one is strongly decreasing, i.e., the amplitude $\left|\mu_{4}\right|$ is very small. This means that the third wave mode should be kept, while the subsequent modes may be ignored.

Consider the case when the whole structure is partitioned into a left periodic structure made up of $N-1=14$ substructures, and a right extra substructure. Assume that the periodic structure is modeled by means of a reduced number $m$ of right/left-going wave modes, while the right extra substructure is modeled by means of the conventional FE method. The related FRF is displayed in Figure 6, when different number $m$ of wave modes are dealt with. As 


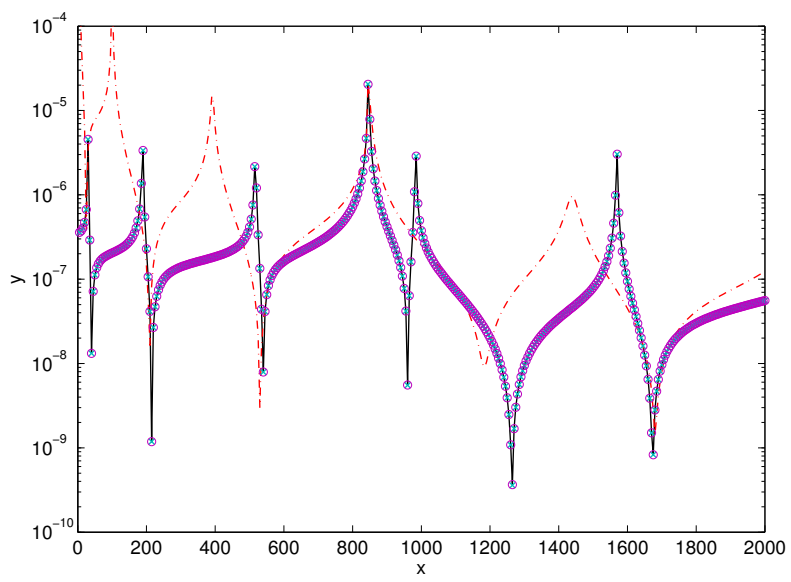

Figure 5: FRF of the 2D periodic structure, as composed of a central periodic structure (13 substructures) surrounded by two extra-substructures: FE solution ( -$)$, WFE solution when the central periodic structure is modeled by means of $m=2$ wave modes $(-\cdot-), m=3$ wave modes $(\times \times \times)$ and $m=4$ wave modes $(\circ \circ \circ)$.

\begin{tabular}{|c|c|c|c|}
\hline$\left|\mu_{1}\right|$ & $\left|\mu_{2}\right|$ & $\left|\mu_{3}\right|$ & $\left|\mu_{4}\right|$ \\
\hline 0.999876 & 0.999704 & 0.387754 & 0.007128 \\
\hline
\end{tabular}

Table 1: Modulus of the first propagation constants, at $2000 \mathrm{~Hz}$.

shown in Figure 6, the WFE solution doesn't match the reference FE curve even though half the wave modes are kept (i.e., $m=11$ ). Convergence is reached when all the wave modes are kept, only. This highlights the fact that near the excitation source, almost the full wave mode basis is needed to recover the dynamic behavior of the system.

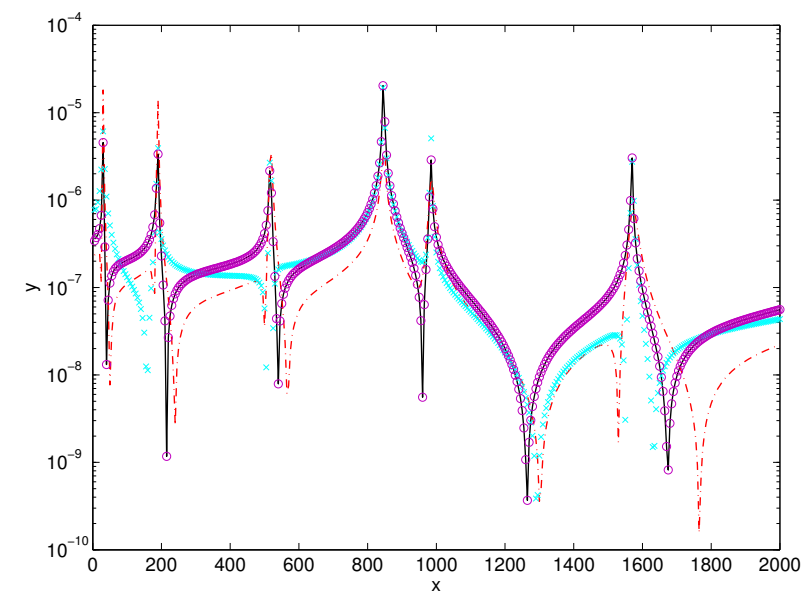

Figure 6: FRF of the 2D periodic structure, as composed of a left periodic structure (14 substructures) coupled with a right extra substructure: FE solution (-), WFE solution when the left periodic structure is modeled by means of $m=3$ wave modes $(-\cdot-), m=11$ wave modes $(\times \times \times)$ and $m=n=22$ wave modes $(\circ \circ \circ)$. 


\subsection{D periodic structure}

As a second example, the harmonic behavior of a complex 3D periodic structure is analyzed by means of the WA approach (Section 3.2.2). The structure under concern consists in a stiffened cylindrical shell, as shown in Figure 7. It is composed of $N=20$ identical substructures, each of these being made up of eighteen longitudinal flat stiffeners and one circumferential L-shaped stiffener. The cylindrical shell and the stiffeners share the following characteristics:

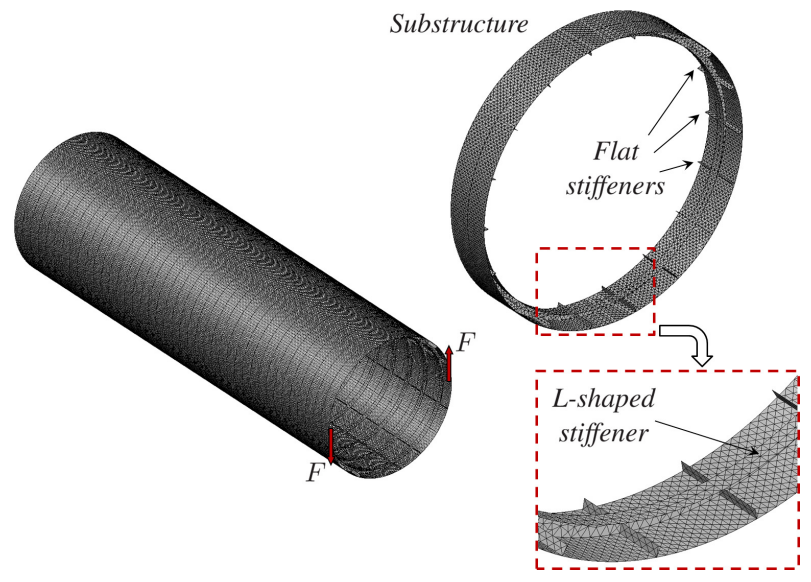

Figure 7: FE model of a stiffened cylindrical shell composed of 20 substructures.

Young's modulus of $70 \mathrm{GPa}$, density of $2700 \mathrm{~kg} / \mathrm{m}^{3}$, Poisson's ratio of 0.3 , loss factor of 0.005 , thickness of $0.002 \mathrm{~m}$. The length and the external diameter of the whole structure are $10 \mathrm{~m}$ and $3 \mathrm{~m}$, respectively. Also, the longitudinal stiffeners have a height of $0.05 \mathrm{~m}$, while the circumferential L-shaped stiffener has a height and a width of $0.1 \mathrm{~m}$. The substructures are meshed in a same manner, i.e., by means of 2D triangular shell elements with three nodes and six DOFs per node that incorporate 3D displacements/rotations. As a whole, each substructure is meshed using 32, 616 DOFs, which are partitioned into $n=1188$ DOFs on each left/right cross-section, and 30, 240 internal DOFs. Also, the total number of DOFs used to model the whole periodic structure is 629,748 , which appears to be large.

Besides, the periodic structure is subject on its left end to vertical point forces of magnitude $F=1000 N$ acting in opposite directions on the cross-section DOFs of two diametrically opposed longitudinal stiffeners (Figure 7). Regarding the right end of the periodic structure, the cross-section DOFs of the longitudinal stiffeners are translationally fixed and rotationally free, while the cross-section DOFs of the cylindrical shell are free. The FRF of the periodic structure is estimated using 300 discrete frequencies which are equally spaced over the frequency band $[0.5 \mathrm{~Hz}, 150 \mathrm{~Hz}]$. Prior to the computation of the wave modes, the condensed dynamic stiffness matrix $\mathbf{D}^{*}$ of a substructure needs to be assessed at each discrete frequency involved within the frequency band of concern. This is achieved using the CB method (cf. Section 3.1), by considering 1000 fixed interface modes of the substructure. The computation of the wave modes follows by considering the eigenproblem (6). The Lanczos method enables one to compute a reduced set of wave modes, only. For this purpose, the WA method proposed in Section 3.2.2 is considered along with the wave mode selection process proposed in Section 3.3. Here, the number of retained wave modes is determined when the value of the error bound $\mathcal{E}_{s}$ shrinks under a tolerance threshold of $10 \%$. This provides $m=125 \mathrm{right} / \mathrm{left}$-going wave modes to be retained, which represents almost $10 \%$ of the total number $n=1188$ of wave modes involved when the eigenproblem (6) is solved directly. 
The FRF of the periodic structure is displayed in Figure 8. Here, the magnitude of the radial displacement is assessed at a measurement point located $1.5 \mathrm{~m}$ far from the excitation sources, along the longitudinal direction. The solution issued from the WA approach (Section 3.2.2) is computed using MATLAB ${ }^{\circledR}$ and compared to the solution issued from a conventional FE software. As it can be seen, the WFE and FE solutions (black and violet curves) perfectly match to each other, hence giving credit to the proposed approach. In terms of CPU times, it takes around $5826 s$ with the WFE method to compute the FRF of the structure using MATLAB ${ }^{\circledR}$ and an Intel ${ }^{\circledR}$ Core $^{\mathrm{TM}}$ i7-3720QM processor, against $13,618 s$ with a commercial FE software and the same processor. This means $57 \%$ time savings in benefit of the WFE method.

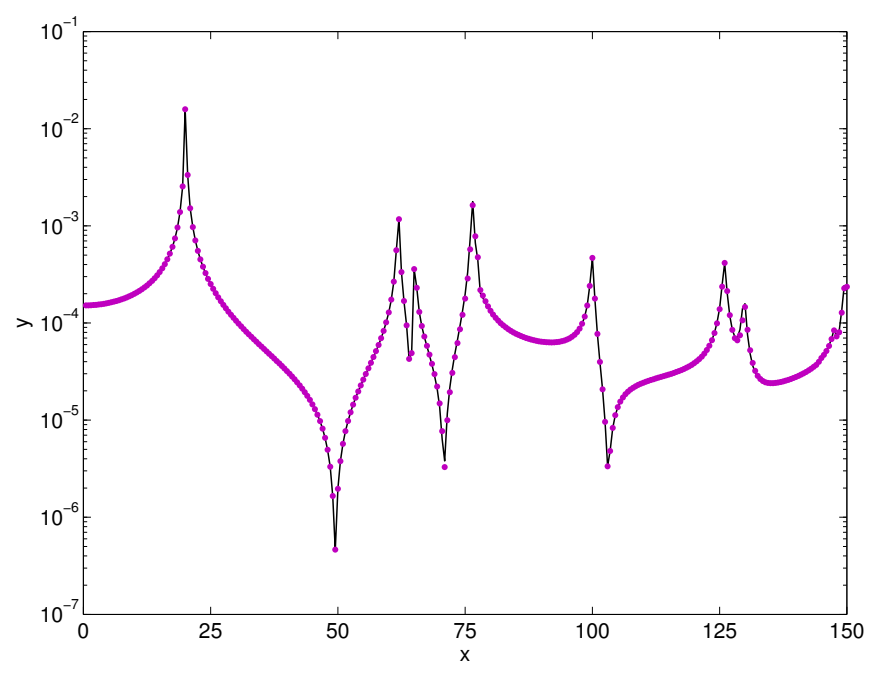

Figure 8: FRF of the stiffened cylindrical shell: FE solution (-) and WFE solution when the central periodic structure is modeled by means of $m=125$ wave modes $(\bullet \bullet \bullet)$.

\section{Concluding remarks}

A model reduction technique has been developed within the framework of the WFE method for the description of the dynamic behavior of structures with one-dimensional periodicity. An efficient generalized eigenproblem based on the so-called $\mathbf{S}+\mathbf{S}^{-1}$ transformation has been proposed for computing the wave modes traveling in right and left directions along a periodic structure composed of arbitrary-shaped substructures. An error indicator has been proposed for selecting in an a priori process those wave modes which mostly contribute to the structure dynamics. It has been shown that the efficiency of the procedure can be greatly improved by partitioning the whole periodic structure into one central structure surrounded by two extra substructures. The feature of this technique lies in the use of a few wave modes for modeling the central structure, hence enabling the CPU times associated to the computation of the WFE eigenproblem to be largely decreased. The relevance of this reduction technique has been clearly validated regarding a 2D periodic structure. Also, it has been successfully applied to model a 3D periodic stiffened shell that contains several substructures with large-sized FE models. 


\section{REFERENCES}

[1] D. Mead, A general theory of harmonic wave propagation in linear periodic systems with multiple coupling, Journal of Sound and Vibration 27 (2) (1973) 235-260.

[2] W. X. Zhong, F. W. Williams, On the direct solution of wave propagation for repetitive structures, Journal of Sound and Vibration 181 (3) (1995) 485-501.

[3] D. Duhamel, B. Mace, M. J. Brennan, Finite element analysis of the vibrations of waveguides and periodic structures, Journal of Sound and Vibration 294 (1-2) (2006) 205-220.

[4] J.-M. Mencik, On the low- and mid-frequency forced response of elastic systems using wave finite elements with one-dimensional propagation, Computers and Structures 88 (1112) (2010) 674-689.

[5] J. Renno, B. Mace, On the forced response of waveguides using the wave and finite element method, Journal of Sound and Vibration 329 (26) (2010) 5474-5488.

[6] Y. Waki, B. Mace, M. Brennan, Numerical issues concerning the wave and finite element method for free and forced vibrations of waveguides, Journal of Sound and Vibration 327 (1-2) (2009) 92-108.

[7] J.-M. Mencik, A wave finite element-based formulation for computing the forced response of structures involving rectangular flat shells, International Journal for Numerical Methods in Engineering 95 (2) (2013) 91-120.

[8] J.-M. Mencik, New advances in the forced response computation of periodic structures using the wave finite element (WFE) method, Computational Mechanics 54 (3) (2014) 789-801.

[9] R. R. Craig, M. C. C. Bampton, Coupling of substructures for dynamic analyses, AIAA Journal 6 (7) (1968) 1313-1319.

[10] G. J. Golub, C. F. V. Loan, Matrix Computations, The Johns Hopkins University Press, Baltimore and London, 1996.

[11] J.-M. Mencik, A model reduction strategy for computing the forced response of elastic waveguides using the wave finite element method, Computer Methods in Applied Mechanics and Engineering 229-232 (2012) 68-86.

[12] J.-M. Mencik, M. N. Ichchou, Multi-mode propagation and diffusion in structures through finite elements, European Journal of Mechanics - A/Solids 24 (5) (2005) 877-898.

[13] R. Patel, On computing the eigenvalues of a symplectic pencil, Linear Algebra and its Applications 188-189 (1993) 591-611.

[14] W.-W. L. T.-M. Huang, J. Qian, Structure-preserving algorithms for palindromic quadratic eigenvalue problems arising from vibration of fast trains, SIAM Journal on Matrix Analysis and Applications 30 (4) (2009) 1566-1592. 